\title{
El retorno de las víctimas como condición implícita para la reparación: las limitaciones de la Ley de Víctimas y Restitución de Tierras
}

Chrysalide Duarte Castro

Felipe Aliaga Sáez

\section{Introducción}

$\mathrm{Z}^{1}$ prolongado conflicto armado en Colombia ha dejado innumeCrables consecuencias, una de las más trágicas son los más de siete millones de víctimas de desplazamiento interno, así como el fenómeno de las personas desplazadas transfronterizas que huyen del país en busca de mejores oportunidades, o por lo menos en busca de preservar su vida, las cuales están cerca al medio millón de personas, la cifra varía significativamente de acuerdo con la fuente de consulta. En las últimas décadas, Colombia ha experimentado diversas reformas normativas e institucionales, donde la justicia ha sido la protagonista en la búsqueda, supuestamente, de encontrar soluciones a las graves violaciones a los derechos humanos. Uno de estos intentos por reparar esas violaciones sistemáticas a los derechos humanos es la Ley 1448, Ley de Víctimas y Restitución de Tierras: por la cual se dictan medidas de atención, asistencia y reparación integral a las víctimas del 
conflicto armado interno y se dictan otras disposiciones, aprobada por el Congreso de la República en el 2011.

La Ley 1448 fue "pensada como engranaje en la política transicional que facilite el paso hacia un escenario de posconflicto, esta ley enuncia como objetivo principal el reconocimiento de las víctimas del conflicto armado colombiano [...] y de sus derechos a la verdad, la justicia y a la reparación, con garantías de no repetición" (Martínez, 2013, p. 3). La Ley de Víctimas y Restitución de Tierras se convirtió en un proyecto ambicioso, reconocido y aplaudido por muchos tanto a nivel nacional como internacional, la propia ley lo evidencia cuando cita las palabras de Norbert Wühler, jefe del Programa de Reparación a nivel mundial de la Organización Internacional para las Migraciones, al referirse a esta política como "el programa más ambicioso e integral de reparación que se haya visto en el mundo entero" (2011, p.18).

No es la intención de este capítulo desconocer los avances que se han presentado en el ámbito del reconocimiento de los derechos jurídicos y políticos de las víctimas del conflicto armado en Colombia. No obstante, y a pesar de la promulgación de esta ley, las víctimas siguen siendo silenciadas y son un problema de enormes magnitudes en Colombia, en donde la mayoría de estas personas no tendrán derecho a una reparación adecuada, si se puede hablar de reparación al sufrir las atrocidades del prolongado conflicto colombiano.

Al hablar de invisibilidad de las víctimas internas, la invisibilidad de las víctimas en el exterior es aún peor, esta problemática no ha cambiado y aun cuando son víctimas del conflicto armado interno no se les ha dado la palabra, son víctimas sin rostro que han tenido que dejar todo atrás. En otras palabras, las víctimas que se encuentran en el exterior, especialmente aquellas en necesidad de protección internacional, son oprimidas casi de manera exponencial. Por un lado, son desplazados por la violencia y son víctimas transfronterizas; y por otro lado, se enfrentan a situaciones de discriminación en los países receptores.

Casi cuatro años después de la implementación de la Ley de Víctimas, Angélika Rettberg hace un balance, en el cual sostiene que la ley goza de un amplio consenso y apoyo popular en torno a su validez moral y política; pero, por otro lado, enfrenta grandes retos como 
la sostenibilidad fiscal, la voluntad de los colombianos de asumir el costo de reparación — no solo de las víctimas- sino del proceso de paz, la capacidad de respuesta institucional, las organizaciones que defienden los derechos de las víctimas como un nuevo actor en la escena política nacional, y finalmente se debe pensar si, en algún momento, las víctimas se van a sentir reparadas (Rettberg, 2015). En el análisis es importante agregar las afectaciones negativas que esta Ley tiene sobre algunas víctimas, especialmente las que se encuentran en necesidad de protección internacional, pues si bien están contempladas en la Ley, solo se hace de manera somera y los mecanismos de reparación y asistencia son poco realistas para las víctimas que se encuentran en el exterior.

Es en este sentido que este capítulo adquiere relevancia, en tanto pretende demostrar, por un lado, las limitaciones que tiene la Ley 1448 para que las víctimas colombianas residentes en el exterior puedan acceder a las medidas de asistencia y reparación que ofrece dicha Ley, y por el otro el retorno de las víctimas como condición implícita para la reparación.

\section{Las limitaciones en la aplicación de la Ley de Víctimas y Restitución de Tierras para las víctimas en el exterior}

Como se mencionó en la introducción, la Ley de Víctimas ha sido elogiada tanto a nivel nacional como internacional, por tratarse de un esfuerzo sin precedentes en el ámbito del reconocimiento de los derechos de las víctimas que ha generado el prolongado conflicto armado en Colombia. Sin embargo, esta Ley puede influir de manera negativa sobre algunas víctimas; el objetivo de este apartado es analizar ese impacto negativo.

En primer lugar, es importante resaltar que en la Ley de Víctimas, con 110 páginas y 208 artículos, solo se hace referencia en tres ocasiones a las víctimas que residen por fuera del territorio colombiano, y cuando se mencionan se hace de manera superflua, sin diferenciar las víctimas que se encuentran en necesidad de protección internacional. En la primera parte del documento se hace referencia a las víctimas en 
el exterior, pero no se hace como parte de su articulado, es solamente una de las diez preguntas más frecuentes acerca de la Ley 1448, específicamente es la pregunta número ocho que plantea lo siguiente, "¿cómo protege a la víctima que se encuentra asilada en el exterior?", a lo cual se responde:

La Ley de Víctimas y Restitución de Tierras establece una serie de herramientas para garantizar que las víctimas que se encuentran fuera del territorio nacional con ocasión del conflicto armado interno sean incluidas en los programas de retorno y de reubicación previstos en la Ley.

Una vez hayan retornado las personas que se encuentran fuera del territorio nacional podrán acceder a las medidas de asistencia y reparación previstas en la Ley, para lo cual deberán presentar la solicitud de inclusión en el Registro Único de Víctimas (Ley 1448, p. 17).

Al respecto de los programas de retorno y reubicación, el artículo 66 estipula que la Unidad Administrativa Especial para la Atención y Reparación a las Víctimas se encargará de que las víctimas de desplazamiento forzado que se encuentran en el exterior sean incluidas en los planes de retorno y reubicación que estipula dicho artículo. Pero cabe anotar que el artículo 66 no contempla planes de retorno, solo hace mención al deber que tiene la unidad administrativa arriba mencionada para adelantar gestiones ante las distintas entidades que conforman el Sistema Nacional de Atención y Reparación a las Víctimas con el fin de brindar una atención integral a la población retornada o reubicada, en los ámbitos de vivienda, educación, salud y orientación laboral (Ley 1448, art. 66); sin embargo, no se estipula de qué manera se llevarán a cabo esas medidas y mucho menos se evidencia un plan de retorno o reubicación. Posteriormente, en el artículo 73 se establece que "las víctimas del desplazamiento forzado y del abandono forzado, tienen derecho a un retorno o reubicación voluntaria en condiciones de sostenibilidad, seguridad y dignidad (Ley 1448, art. 73). Hasta el momento, la primera referencia a las 
víctimas en el exterior presupone que la reparación de las mismas está condicionada al retorno.

La segunda referencia a las víctimas en el exterior se encuentra en el artículo 149, concerniente a las garantías de no repetición, en el inciso J del mismo se indica que se hará "difusión de la información de los derechos de las víctimas radicadas en el exterior" (Ley 1448, p. 80). Nuevamente, no se ahonda en una explicación que determine por medio de qué mecanismos se llevará a cabo la difusión, y los testimonios de algunas víctimas en el exterior sugieren que la difusión de la información, y particularmente de la Ley de Víctimas, ha sido muy precaria, y en algunos casos nula. Finalmente, el artículo 204 dicta que "el Gobierno nacional, a través del Ministerio de Relaciones Exteriores, y de acuerdo con lo dispuesto en el artículo 30, garantizará que las víctimas de quienes trata la presente ley que se encuentren fuera del país sean informadas y orientadas adecuadamente acerca de sus derechos, medidas y recursos" (Ley 1448, p. 108), en términos prácticos no es muy diferente a lo dispuesto en el artículo 149.

Que las medidas adoptadas para las llamadas víctimas en el exterior sean inadecuadas y por demás restrictivas para este grupo, genera una serie de limitaciones para que estas personas puedan acceder a las medidas de reparación que ofrece la Ley de Víctimas. A pesar de que recientemente el tercer sector se ha preocupado por analizar temas referentes a la reparación de los refugiados, se ha concentrado más en hacerlo en el contexto de escenarios de postconflicto como parte esencial de los procesos de justicia transicional, sin embargo, en Colombia aún no se puede hablar de un posconflicto, pues si bien se negoció un acuerdo de paz con las Fuerzas Armadas Revolucionarias de Colombia - Ejército del Pueblo (Farc-EP) existen otros actores que también son parte importante del conflicto armado en Colombia y que aún siguen activos. A pesar de lo anterior, difícilmente se encuentra información acerca de la reparación de las víctimas en el exterior, por lo cual, para analizar dichas limitaciones se tomará como referencia el trabajo adelantado por la Comisión Colombiana de Juristas (2012), con el apoyo del Consejo Noruego para Refugiados, debido a que es de los pocos trabajos que se han encontrado referentes a la situación particular de las víctimas en el exterior, en el marco de la Ley 1448. 


\section{Las medidas de asistencia y reparación contempladas en la Ley de Víctimas y Restitución de Tierras}

La Ley de Víctimas contempla unas medidas de asistencia y reparación para las víctimas del conflicto armado en Colombia, y debido a que en este documento se sostiene que las víctimas en el exterior difícilmente pueden acceder a estas medidas a causa de las limitaciones que se presentan en dicha ley, se considera importante mencionar brevemente en qué consisten las medidas de asistencia y reparación, para entender de manera más clara cómo se ven afectadas estas víctimas al no poder beneficiarse de estas medidas. En primer lugar, se debe aclarar que las medidas de asistencia son diferentes a las medidas de reparación, las primeras hacen referencia a la asistencia funeraria, en salud, en educación y a ayudas humanitarias, mientras que las segundas están dirigidas a restituciones e indemnizaciones. En la Ley de Víctimas se hace un mayor énfasis en las medidas del segundo tipo, por lo cual se procederá a describir de manera concreta cada una de estas medidas.

La primera medida de reparación hace referencia a la restitución de tierras, a la cual tienen derecho las víctimas que eran propietarias de tierras y que fueron obligadas a abandonarlas; de no ser posible la restitución del terreno debido a diversas razones, la víctima podrá solicitar la restitución de un inmueble similar al que perdió. La segunda medida de reparación hace alusión a la restitución de los derechos sociales, que consisten en la priorización en los subsidios de vivienda, beneficios para pago de créditos y generación de programas de empleos e ingresos. En tercer lugar se encuentra la indemnización de carácter administrativo, que corresponde a una indemnización monetaria, de acuerdo con el hecho victimizante, el orden de entrega de estas indemnizaciones se hará conforme a unos criterios de priorización.

La Ley de Víctimas también contempla la implementación de medidas de rehabilitación que consisten en el restablecimiento de las condiciones físicas y psicológicas. Otro tipo de medidas son las de satisfacción, son medidas enfocadas a la reparación simbólica de las víctimas, a la construcción de memoria histórica, a 
reconocimientos públicos, hasta el juzgamiento de los victimarios. Dentro de estas también se encuentran las garantías de no repetición, que incluyen varias medidas, partiendo de la construcción de un país en paz, el objetivo principal es garantizar que no se repitan los hechos victimizantes por los que atravesaron las víctimas. Por último, se encuentran las medidas de reparación colectiva que están dirigidas a las violaciones de derechos colectivos o a miembros de grupos establecidos ${ }^{1}$.

\section{Las limitaciones en la aplicación de la Ley de Víctimas y Restitución de Tierras para los Refugiados Colombianos}

El objetivo de este apartado es analizar las limitaciones a la aplicación que tiene la Ley de 1448 para que las víctimas en el exterior puedan acceder a las medidas de asistencia y reparación que ofrece dicha ley, para lo cual se identificaron cuatro tipos de limitaciones, a saber: jurídicas, administrativas, política y psicosociales.

\section{Limitaciones jurídicas}

Con limitaciones jurídicas se hace referencia en primer lugar a la viabilidad que tiene la Ley 1448 para que las víctimas en el exterior puedan, en efecto, acceder a las medidas de asistencia y reparación; y en segundo lugar, evidenciar las contradicciones que la Ley podría tener en torno al marco jurídico internacional en materia de protección de las persona en condición de refugio. El hecho de que las víctimas en el exterior deban presentar su declaración ante los consulados colombianos se convierte en una limitación de índole jurídica, pues sin este trámite las víctimas no pueden acceder a las medidas de reparación,

1 La información ampliada de estas medidas se puede consultar en la Ley de Víctimas, o en el capítulo 2 de un documento de la Unidad para las Víctimas, que se realizó en el marco de "Participaz, la ruta de los derechos" como una estrategia para visibilizar los derechos de las víctimas. Dicho documento se tomó como referencia para redactar este párrafo, el cual, a 6 de mayo de 2017, se encontraba disponible en el siguiente enlace: http://participaz.com/images/ cartillas/Capitulo_2.pdf. 
lo cual se puede entender como una inconsistencia de índole jurídica ya que el texto de la Ley no contempla de manera adecuada las condiciones y necesidades de las víctimas refugiadas.

En la primera limitación, si bien la Ley cobija a las víctimas que hayan huido del país a causa del conflicto armado, esto no implica necesariamente que las mismas puedan acceder a las medidas de reparación, no solo por razones de índole administrativa como se verá en el siguiente apartado, sino porque jurídicamente para realizar algunos trámites el gobierno colombiano exige la presencia física de la víctima. Por ejemplo, en los casos donde aplique la restitución de tierras, cuyo trámite de inscripción de los predios es igual de inconveniente que el del Registro Único de Víctimas (RUV) —como se verá en el apartado de las limitaciones administrativas-, y suponiendo que se lograra superar el obstáculo de la inscripción del predio, surge otra limitación relacionada con que "el marco normativo dispuesto hasta ahora no brinda herramientas para que las PNPI $^{2}$ tramiten sus demandas en territorio colombiano, sin necesidad de tener que realizar trámites en Colombia que le impliquen regresar al país, así sea de manera temporal, para realizar diligencias judiciales" (Comisión Colombina de Juristas, 2012, pp. 26-27).

La segunda limitación gira en torno a las contradicciones que podría presentar la Ley al realizar un análisis desde el derecho internacional, lo cual, en vez de ser positivo, podría ser contraproducente para los refugiados que se cobijen con las medidas de asistencia y reparación que ofrece la Ley, en tanto que podría poner en riesgo la protección internacional de la que gozan por el hecho de contar con el estatuto de refugiados. En este sentido, conviene resaltar que Colombia ha firmado y ratificado los instrumentos internacionales más importantes en materia de protección a personas refugiadas, incluyendo la Declaración de Cartagena de 1984, la cual juega un papel importante en este punto, pues se puede correr el riesgo de que "la definición del universo de víctimas contenida en la Ley 1448 (art. 3) pudiera ser interpretado como un retroceso frente a la definición

2 Esta sigla hace referencia a "personas en necesidad de protección internacional". 
ampliada de la Declaración de Cartagena" (Comisión Colombiana de Juristas, 2012, p. 4), la cual es considerada como un gran aporte desde América Latina en el ámbito del derecho internacional para la protección de los refugiados, teniendo en cuenta el contexto particular de la región, en este sentido:

Un aporte fundamental de la Declaración de Cartagena ha radicado también en la consagración de la definición regional del término refugiado que complementa la definición prevista en el artículo 1 de la Convención sobre el Estatuto de los Refugiados de 1951 y que reconoce la necesidad de extender el alcance de la protección internacional a «las personas que han huido de sus países porque su vida, seguridad o libertad han sido amenazadas por la violencia generalizada, la agresión extranjera, los conflictos internos, la violación masiva de los derechos humanos u otras circunstancias que hayan perturbado gravemente el orden público».

A pesar de constituir un instrumento de carácter no vinculante para los Estados por tratarse de una declaración, su enfoque innovador y el amplio reconocimiento que le han otorgado a este instrumento regional los Estados de la región han convertido a la Declaración de Cartagena sobre Refugiados de 1984 en una guía fundamental para el diseño y la evaluación de las políticas regionales en materia de protección de refugiados (Demant, 2013, pp. 131-132).

En conclusión, por un lado la definición de víctima adoptada en el artículo tercero de la Ley 1448 podría generar que los Estados receptores no reconozcan el estatuto de refugiado a personas que hayan huido del país por razones diferentes al conflicto armado interno; y por otro lado, algunos Estados están interpretando la Ley de Víctimas como una medida de reparación en sí misma y, en ese sentido, si los refugiados se acogen a las medidas de asistencia y reparación, se podría interpretar como la desaparición de las causantes de solicitud de refugio y por ende de la necesidad de protección internacional (Comisión Colombiana de Juristas, 2012, pp. 5-6). 


\section{Limitaciones administrativas}

Otro tipo de limitaciones que se han evidenciado en la Ley se pueden agrupar de cierto modo en la categoría de administrativas; por tratarse de limitaciones de índole administrativa no deben considerarse menos importante que otro tipo de limitaciones, por el contrario, estas restricciones son tan importantes que pueden ocasionar que los refugiados ni si quiera puedan inscribirse en el Registro Único de Víctimas, que es uno de los requisitos para poder acceder a las medidas de asistencia y reparación de la Ley.

Existe una cartilla elaborada por el Ministerio de Relaciones Exteriores que tiene como título Orientaciones generales para colombianos/as victimas en el exterior sobre el acceso a medidas de atención, asistencia y reparación en el marco de la Ley 1448 de 2011. A primera vista parece ser un documento claro en el que gobierno colombiano no solo reconoce la existencia de las víctimas en el exterior, sino que además invita a que las mismas conozcan las acciones que adelanta el Gobierno para garantizar las medidas de atención y reparación en palabras textuales, la cartilla dice lo siguiente:

En las últimas décadas, miles de personas se han visto obligadas a salir del país por razones vinculadas directamente con el conflicto armado interno colombiano. Por ello, hoy el Estado colombiano reconoce a las víctimas que se encuentran en el exterior como parte del universo de víctimas del conflicto armado y realiza los esfuerzos necesarios para que su reparación se convierta en realidad.

Ese reconocimiento de las víctimas es parte fundamental de la reconciliación como sociedad. Sabemos que han tenido que pasar por muchos momentos difíciles y hoy les decimos que no están solos, no están solas. Por eso, si usted o alguien de su familia ha sufrido un daño como consecuencia de las violaciones de que trata el artículo 3 de la Ley 1448, es importante que conozcan las acciones que adelanta el Estado colombiano para garantizarles las medidas de atención y reparación, cuya entrega sea posible en el exterior, así como ser parte del diseño de programas especiales de acompañamiento para quienes voluntariamente deseen 
retornar a territorio nacional (Orientaciones para colombianos víctimas en el exterior).

Por lo anterior, en el documento se indica que el Estado amplía el proceso de inclusión en el RUv a las víctimas residentes en el exterior. Hasta aquí todo parece razonable, sin embargo, los problemas se empiezan a evidenciar más adelante, en los apartados segundo y tercero de esta cartilla. El segundo apartado está dedicado a los colombianos con protección internacional, punto en el cual se sostiene que, en principio, acceder a las medidas de reparación que ofrece la Ley 1448 no debería afectar la protección internacional de la que gozan las personas con este tipo de protección; pero que deberá consultarse en cada caso con el país receptor si beneficiarse de esas medidas podría tener repercusiones sobre su estatuto. En esta misma sección se indica que la inscripción en el RUV deberá tramitarse ante el Consulado del país donde vivan, así como la inscripción para la restitución de tierras en los casos en los que aplique.

¿Por qué se afirma que estas disposiciones presentan limitaciones para las víctimas en el exterior? En el primer caso, "muchos estados receptores consideran que la tramitación de cualquier diligencia que los refugiados realicen ante las embajadas o consulados, siendo estos representantes del Estado que no puede brindarles protección, es una aceptación tácita del refugiado de que su Estado sí está en capacidad de brindarle protección" (Comisión Colombiana de Juristas, 2012, p. 18), en este sentido, los refugiados podrían abstenerse de presentar su declaración para la inscripción en el RUv, por miedo a que su estatuto como refugiados se vea afectado, ocasionando que no puedan acceder a las medidas de asistencia y reparación que ofrece la Ley de Víctimas, en tanto que este registro es un requisito fundamental. En el segundo caso, el que se obligue a las víctimas refugiadas a presentar sus declaraciones ante el Consulado de Colombia presenta, por lo menos, tres limitaciones: en primer lugar, no existe presencia consular de Colombia en todos los países donde residen víctimas colombianas y, en los países donde existen, normalmente están ubicados en las capitales y por razones económicas muchas víctimas no tienen los medios para desplazarse hasta los consulados; en segundo lugar, "el 
hecho de que las embajadas y consulados, que representan al Estado colombiano en territorio internacional, sean quienes reciban las solicitudes de las víctimas en materia de registro, es problemático porque puede ser interpretado por los estados de acogida como un hecho indicativo de que la persona se acogió voluntariamente a la protección de su país de nacionalidad" (Comisión Colombiana de Juristas, 2012, pp. 17-18). Por último, algunas víctimas en el exterior, en especial las que han huido del país a causa de la persecución de su propio Estado, no quieren tener contacto con las instituciones estatales por miedo a posibles represalias.

\section{Las limitaciones políticas}

Las limitaciones de este tipo son importantes pero desafortunadamente la información al respecto es supremamente limitada, por no decir nula. Sin embargo, es necesario mencionarlas pues la falta de voluntad política en Colombia ha generado, en gran medida, que la problemática de las víctimas en el exterior no sea considerada como una situación que amerite la creación de políticas o programas de gobierno. Es precisamente la falta de voluntad política la que ha dado la espalda a este grupo de víctimas y el texto de la Ley de Víctimas refleja eso, que no existe un verdadero interés político por hacer frente a los miles de refugiados dispersos en diferentes países, probablemente en un escenario de posconflicto en Colombia este tema pueda resultar de importancia para algún partido político o incluso convertirse en un tema de interés nacional al advertir que "Colombia se ubicó entre los 10 países que más refugiados nacionales tiene a nivel mundial; categoría en la que ocupa el primer lugar a nivel latinoamericano" (El Heraldo, 2016), o probablemente, al igual que sucedió con la Ley de Víctimas, eventualmente podría servir como bandera política de algún gobierno, de pronto en un escenario de posconflicto donde se logre dar solución no solo a la situación de las millones de víctimas al interior del país sino también a las miles de víctimas que se encuentran en el exterior.

Las críticas al Estado colombiano también se dan desde los países receptores, por ejemplo, Alfonso Morales, director de refugiados de la Cancillería de Ecuador, como país que alberga el mayor número de 
refugiados colombianos, ha criticado "la 'falta de voluntad política' del gobierno colombiano para entregar un aporte financiero para la atención a los refugiados en Ecuador" (Mena, 2010).

\section{Limitaciones psicosociales}

Este tipo de limitaciones incluyen factores que, comúnmente, no se contemplan como las más importantes a la hora de pensar en las reparaciones a las víctimas; normalmente se hace énfasis en reparaciones de tipo económico o de restitución de tierras. Si bien la Ley de Víctimas contempla en su articulado la reparación de tipo psicosocial, es pertinente analizar de qué manera se entiende ese concepto y especialmente su viabilidad en la aplicación a las víctimas refugiadas. Para lo anterior, vale la pena estudiar tanto la definición dada en la Ley, como la de algunos autores en el contexto académico, para determinar qué tipo de limitaciones podrían presentarse para que los refugiados accedan a este tipo de medidas de reparación. En cuanto a la reparación psicosocial es importante destacar que en la Ley de Víctimas se evidencia un esfuerzo por dar respuesta a este tipo de atención, sin embargo, como pasa con las otras medidas de asistencia y reparación, difícilmente estas medidas pueden ser aplicadas a las víctimas refugiadas.

De acuerdo con la Ley, "la atención psicosocial debe ser entendida como los procedimientos de acompañamiento que promuevan la recuperación de la salud mental de los individuos y la reconstrucción de las bases fundamentales de sus relaciones sociales" (Ley 1448, p. 16). Dentro de las medidas de rehabilitación como medida de reparación también se tiene en cuenta el restablecimiento de las condiciones psicosociales. La Ley también contempla el apoyo psicosocial como un acompañamiento transversal a todo el proceso de reparación:

El acompañamiento psicosocial deberá ser transversal al proceso de reparación y prolongarse en el tiempo de acuerdo con las necesidades de las víctimas, sus familiares y la comunidad, teniendo en cuenta la perspectiva de género y las especificidades culturales, religiosas y étnicas. Igualmente debe integrar a los familiares y de ser posible promover acciones de discriminación positiva a favor 
de mujeres, niños, niñas, adultos mayores y discapacitados debido a su alta vulnerabilidad y los riegos a los que se ven expuestas (Ley 1448, art. 136).

Adicionalmente, se decreta la creación del Programa de Atención Psicosocial y Salud Integral de Víctimas, el cual, de acuerdo con el Informe del Gobierno nacional a las Comisiones Primeras del Congreso de la República, del segundo semestre de 2013 y a la página del Ministerio de Salud de Colombia, ya se encuentra en funcionamiento. No obstante, ni en la Ley, ni en el citado informe, ni en la página del Ministerio se hace referencia a la atención psicosocial de las víctimas en el exterior. Es más, "el Ministerio de Salud y Protección Social no cuenta con herramientas para darle una extensión territorial de aplicación a esta ley [la de víctimas], y, por lo tanto, no puede brindar atención psicosocial a las víctimas que se encuentran residiendo en el exterior", dice el Gobierno en uno de esos informes (El Tiempo, 2015). Esto se complejiza aún más debido a que en el Documento Conpes 3712, por medio del cual se adopta el plan de financiación de la Ley 1448 de 2011, no se designan recursos para la atención psicosocial de las víctimas en el exterior, en general, y mucho menos de las víctimas refugiadas, en particular.

Como la definición de lo psicosocial se queda corta en la Ley, es pertinente analizar algunos planteamientos un poco más amplios, para entender de qué manera se ven afectadas las víctimas refugiadas al tener limitaciones para acceder a este tipo de reparación. Al respecto, Liz Arévalo considera que:

[...] Lo psicosocial es irreverente frente a procesos de atención puramente psicológicos y focalizados en los individuos, e invita a adelantar acciones tendientes a integrar lo emocional y lo relacional con una comprensión desde el contexto. La perspectiva psicosocial permite comprender, así, la particularidad de la población víctima de la violencia socio-política, re-conocer sus múltiples contextos sociales, culturales y políticos como ámbitos en los que se construye y deconstruye la identidad, el mundo emocional y relacional, los cuales son constituyentes de la realidad que se vive. 
En este marco, consideramos que el acompañamiento psicosocial tiene como objetivo construir un proceso reflexivo entre la población víctima, su red social y los acompañantes, que contribuya a la superación de los efectos sociales y emocionales de la violencia a través de la resignificación de la identidad y del reconocimiento de recursos personales y sociales, en el marco de la categoría de sujeto de derechos (Arévalo, 2010, p.30).

En esta definición se pueden resaltar varios factores importantes para la reparación psicosocial de las víctimas, por un lado, el reconocimiento de la parte emocional relacionada con el contexto social, político y cultural de cada víctima y la importancia de trabajar integralmente para la resignificación de la víctima. Por otro lado, asumir a la reparación psicosocial como algo secundario puede "impedir a las comunidades encontrar salidas que transformen su hoy y ahora, anclándolas en la memoria del dolor y dificultando la reconstrucción de los proyectos de vida" (Rebolledo y Rendón, 2010, p. 41). Las víctimas del conflicto armado, a la par que requieren una reparación material, deberían contar con un proceso transversal de acompañamiento psicosocial para lograr reconstruir o reapropiar su identidad.

Si bien los hechos victimizantes son igualmente traumáticos para las víctimas de desplazamiento forzado, tanto interno como externo, no se debe desconocer que las personas que deben cruzar fronteras para salvaguardar su vida se exponen, en la mayoría de casos, a situaciones de vulnerabilidad más graves, pues no solo han tenido que dejar su país, sino que con él dejan sus familias, sus redes de apoyo, su contexto sociocultural y en muchas ocasiones hasta su idioma. Si los desplazados internos se enfrentan a situaciones inhumanas estando en su propio país se puede deducir qué sucede con los refugiados que han dejado absolutamente todo atrás. La Ley de Víctimas no ofrece medidas de reparación psicosocial para las víctimas en el exterior, menos para aquellas que se encuentran en necesidad de protección internacional y requieren de una ayuda diferencial, como lo plantea la Ley para el caso de otros grupos vulnerables.

A lo largo de este apartado se intentó contextualizar al lector en el entendimiento de la Ley de Víctimas y Restitución de Tierras, a través 
de una aproximación general, pero especialmente a través de las diferentes limitaciones que presenta la aplicación de esta Ley para las víctimas en el exterior. Se identificaron limitaciones de índole jurídica, administrativa, política y psicosocial que afectan negativamente a un grupo de víctimas, y que impiden, o por lo menos dificultan mucho, el proceso para que los refugiados colombianos puedan acceder a las medidas de asistencia y reparación que contempla la Ley. En ese sentido, se entiende que existe una condición implícita del retorno como único mecanismo para garantizar la reparación de las víctimas en el exterior.

\section{Los mecanismos de retorno en la Ley de Víctimas}

Es importante detenerse a analizar brevemente qué ha pasado con los "planes" de retorno y reubicación previstos en la Ley, específicamente para la población refugiada, pues este es uno de los temas más importantes en materia de política migratoria. En primer lugar, la Ley hace alusión exclusivamente al retorno de víctimas de desplazamiento forzado, sin tener en cuenta que muchas víctimas en el exterior tuvieron que salir por hechos victimizantes diferentes al desplazamiento forzado. En ese caso es válido cuestionarse si las víctimas registradas bajo otros hechos no pueden acceder a las medidas de retorno y reubicación. Por otro lado, no se tiene en cuenta el enfoque diferencial que deberían tener las víctimas en el exterior; al encontrarse fuera del territorio nacional el acompañamiento por parte del Estado debería iniciarse desde sus países de residencia, no solo en términos económicos sino psicosociales, analizando los cambios sociales y culturales a los que está expuesta la población que desea retornar.

Normativamente, la Ley de Víctimas no es la única que contempla el retorno, paralelamente a dicha Ley, en junio de 2011, mismo mes y año en que se expidió la Ley de Víctimas, también se expidió la Ley 1465 de 2011, por la cual se crea el Sistema Nacional de Migraciones y se expiden normas para la protección de los colombianos en el exterior. El artículo 8 de dicha Ley establece que por iniciativa parlamentaria o del Gobierno nacional, se formulará un plan de retorno (Ley 1465 de 2011, art. 8). Al siguiente año se sancionó la Ley 1565 de 2012 por medio de la cual se dictan disposiciones y se fijan incentivos para el 
retorno de los colombianos residentes en el extranjero; “si bien [esta] constituye un avance significativo en cuanto al fortalecimiento del retorno de los colombianos que viven en el exterior, puesto que por primera vez la legislación colombiana brinda incentivos y acompañamiento a aquellos conciudadanos que deseen regresar voluntariamente al país, la literatura académica ha evidenciado algunas limitaciones en cuanto a la operatividad de la ley" (Aliaga, 2017):

En la Ley 1565, aprobada el pasado 31 julio, aunque se fijan estímulos para el retorno, priman los incentivos tributarios para la entrada de diverso tipo de bienes y recursos de aquellos retornados que acumularon para su retorno. No obstante, por ejemplo, para quienes perdieron su vivienda al quedar cesantes en España, quienes no lograron acumular capital, o vienen desempleados, los incentivos son pocos. La Ley aprobada no dispone de recursos suficientes para poner en marcha programas ambiciosos que beneficien realmente a los retornados en condiciones precarias y sus familias. Perú, por su parte, ha intentado organizar programas que faciliten la incorporación, especialmente productiva y económica de sus retornados, en cuyo contexto la sociedad civil ha participado en su construcción. En consecuencia, podría afirmarse que las políticas públicas de retorno solo ofrecen un panorama claro para quienes retornan con buenos recursos y capital financiero (Muñoz, Mejía y Castro, 2012, p. 11).

El problema con los planes de retorno no está relacionado con la falta de un marco jurídico, sino con una articulación entre los diferentes instrumentos jurídicos y la voluntad política para que, en efecto, se puedan poner en marcha las medidas contempladas en las diferentes leyes. Adicionalmente, como se indicaba en el párrafo anterior, el marco jurídico está beneficiando a los que se podrían nombrar como retornados deseados, dejando de lado a la gran mayoría de víctimas refugiadas.

\section{Conclusiones}

Teniendo en cuenta el análisis realizado a lo largo de este capítulo en torno a los mecanismos de retorno, se hace evidente que lo planteado 
en el texto de la Ley de Víctimas no responde a las necesidades de las víctimas refugiadas, ni establece el procedimiento por seguir para que los derechos de este grupo de personas se garanticen. En primer lugar, no se enuncian cuáles serán las herramientas que se mencionan en materia de reparación a las víctimas que residen en exterior y, en segundo lugar, existe una condición implícita del retorno como único mecanismo para que este grupo de víctimas pueda acceder a una reparación integral. Lo anterior se puede afirmar al analizar la ruta de reparación en el exterior, la cual es poco realista y aplicable para las víctimas refugiadas; esto implica que para pensar en una reparación, en una restitución de tierras, en un acompañamiento psicológico o en cualquier de las medidas de asistencia y reparación que ofrece la Ley 1448 , las víctimas tendrían que retornar al territorio colombiano, sin tener en cuenta que algunas personas no cuentan con las garantías para regresar, o el conflicto que supone hacerlo porque esto implicaría un nuevo proceso de desarraigo.

Ahora bien, se considera que el proceso histórico por el cual está atravesando Colombia en aras de construir un país en paz es el escenario pertinente para que Colombia no solo reconozca a las víctimas en el exterior, sino que establezca hojas de ruta y mecanismos para que las mismas puedan acceder a las medidas de asistencia y reparación que ofrece la Ley 1448, ya sea que las víctimas quieran retornar a Colombia o deseen quedarse en el país de acogida, pues el retorno no debe ser una condición para la reparación en tanto se estaría atentando contra el principio de la voluntariedad del retorno.

Finalmente, a pesar de que la Ley de Víctimas marcó un hito importante en el reconocimiento de los derechos jurídicos y políticos de las víctimas del conflicto armado en Colombia; las víctimas, especialmente aquellas que residen en el exterior, siguen siendo silenciadas y este es aún un problema de enormes magnitudes en Colombia, donde la mayoría de víctimas no tendrán derecho a una reparación adecuada, y donde el retorno se convierte casi en la única posibilidad de poder acceder a alguna de las medidas de asistencia o reparación que contiene la Ley. 


\section{Referencias bibliográficas}

Aliaga, F., y otros. (2017). Una innecesaria tipología de la migración de retorno. Análisis crítico de la Ley 1565 para el retorno de colombianos en el exterior. (Inédito).

Arévalo, L. (2010). Atención y reparación psicosocial en contextos de violencia sociopolítica: una mirada reflexiva. Revista de Estudios Sociales, 36, 29-39. En línea. Recuperado de: http://revistas.uniandes.edu.co/doi/ pdf/10.7440/res36.2010.02

Comisión Colombiana de Juristas (2012). Refugiados y Ley de Victimas. Reparación para las personas en necesidad de protección internacional: igualdad en el acceso y diferencia en su desarrollo y aplicación. Bogotá: Comisión Colombiana de Juristas. En línea. Recuperado de: http:// www.coljuristas.org/documentos/libros_e_informes/refugiados_y_ley_ de_victimas_2.pdf

Demant, E. (2013). 30 años de la Declaración de Cartagena sobre Refugiados. Avances y desafíos de la protección de refugiados en Latinoamérica. Agenda Internacional. 31, 131-140. En línea. Recuperado de: http:// revistas.pucp.edu.pe/index.php/agendainternacional/article/view/7731

El Heraldo (2016, 15 de septiembre). Los refugiados que ha dejado el conflicto armado en Colombia. (Sec. Política).

El Tiempo (2015, 14 de abril). La difícil situación de víctimas en el exterior. (Sec. Política).

Gobierno de Colombia. (2011). Orientaciones generales para colombianos/as victimas en el exterior sobre el acceso a medidas de atención, asistencia y reparación en el marco de la Ley 1448 de 2011. En línea. Recuperado de: https:/www.cancilleria.gov.co/sites/default/files/guia_orientacionesgeneralesvictimasexterior.pdf

Gobierno de Colombia. (2011). Ley 1448. Ley de Victimas y restitución de tierras: por la cual se dictan medidas de atención, asistencia y reparación integral a las víctimas del conflicto armado interno y se dictan otras disposiciones.

Gobierno de Colombia. (2011). Ley 1465 de 2011. Por la cual se crea el Sistema Nacional de Migraciones y se expiden normas para la protección de los colombianos en el exterior.

Gobierno de Colombia. (2012). Ley 1565 de 2012. Por medio de la cual se dictan disposiciones y se fijan incentivos para el retorno de los colombianos residentes en el extranjero. 
Martínez, P. (2013). Ley de Víctimas y Restitución de Tierras en contexto: Un análisis de las contradicciones entre el modelo agrario y la reparación a las víctimas. Berlín: Forschungs-und Dokumentationszentrum. En línea. Recuperado de: https://www.tni.org/files/download/martinez-ley-de-victimas-web.pdf

Mena, P. (2010, 10 de abril). Ecuador: miles de refugiados en busca de refugio. BBC Mundo. (Sec. América Latina).

Muñoz, J. (2012). En Mejía, W. y Castro, Y. Retorno de migrantes a la Comunidad Andina.(Prol.). Bogotá: Fundación Esperanza.

Rebolledo, O. y Rondón, L. (2010). Reflexiones y aproximaciones al trabajo psicosocial con víctimas individuales y colectivas en el marco del proceso de reparación. Revista de Estudios Sociales, 36, 40-50. En línea. Recuperado de: http://revistas.uniandes.edu.co/doi/pdf/10.7440/res36.2010.03

Rettberg, A. (2015). Ley de Víctimas en Colombia: un balance. Revista de Estudios Sociales, 54, 185-188. En línea. Recuperado de: http://www.scielo. org.co/scielo.php?script=sci_arttextypid=S0123-885X2015000400015 